\title{
The remote handling compatibility analysis of the ITER generic upper port plug structure
}

\author{
D.M.S. Ronden ${ }^{a, *}$, A. Dammann ${ }^{b}$, B. Elzendoorn ${ }^{a}$, T. Giacomin ${ }^{b}$, C. Heemskerk ${ }^{c}$, \\ D. Loesser ${ }^{\mathrm{d}}$, P. Maquet ${ }^{\mathrm{b}}$, J. van Oosterhout ${ }^{\mathrm{a}}$, S. Pak ${ }^{\mathrm{b}}$, C.S. Pitcher ${ }^{\mathrm{b}}$, M. Portales ${ }^{\mathrm{b}}$, \\ M. Proust ${ }^{\mathrm{b}}$, V.S. Udintsev ${ }^{\mathrm{b}}$, M.J. Walsh ${ }^{\mathrm{b}}$ \\ a FOM Institute DIFFER, P.O. Box 1207, 3430 BE Nieuwegein, The Netherlands \\ b ITER Organization, Route de Vinon sur Verdon, 13115 Saint Paul-lez-Durance, France \\ ${ }^{\mathrm{c}}$ Heemskerk Innovative Technology, Merelhof 2, 2172 HZ Sassenheim, The Netherlands \\ d Princeton Plasma Physics Laboratory, Princeton, NJ 08543-0451, United States
}

\section{H I G H L I G H T S}

- We describe the remote handling compatibility of the ITER generic upper port plug.

- Concepts are presented of specific design solutions to improve RH compatibility.

- Simulation in VR of the GUPP DSM replacement indicates possible collisions.

- Specific tooling concepts are proposed for GUPP handling equipment for the hot cell.

\section{A R T I C L E I N F O}

\section{Article history:}

Received 12 September 2013

Accepted 4 March 2014

Available online 8 April 2014

\section{Keywords:}

ITER

Remote handling

Diagnostics

Port plug

\begin{abstract}
A B S T R A C T
The ITER diagnostics generic upper port plug (GUPP) is developed as a standardized design for all diagnostic upper port plugs, in which a variety of payloads can be mounted. Here, the remote handling compatibility analysis (RHCA) of the GUPP design is presented that was performed for the GUPP final design review. The analysis focuses mainly on the insertion and extraction procedure of the diagnostic shield module (DSM), a removable cassette that contains the diagnostic in-vessel components. It is foreseen that the DSM is a replaceable component - the procedure of which is to be performed inside the ITER hot cell facility (HCF), where the GUPP can be oriented in a vertical position.

The DSM removal procedure in the HCF consists of removing locking pins, an M30 sized shoulder bolt and two electrical straps through the use of a dexterous manipulator, after which the DSM is lifted out of the GUPP by an overhead crane. For optimum access to its internals, the DSM is mounted in a handling device. The insertion of a new or refurbished DSM follows the reverse procedure.

The RHCA shows that the GUPP design requires a moderate amount of changes to become fully compatible with RH maintenance requirements.
\end{abstract}

(C) 2014 Elsevier B.V. All rights reserved.

\section{Introduction}

In its role as port integrator, ITER diagnostics division is developing a standardized, generic upper port plug design (GUPP) (Fig. 1). It houses a removable diagnostic shield module (DSM) that is designed to contain the large variety of diagnostic payloads, currently being developed for use in ITER. The diagnostic first wall

\footnotetext{
* Corresponding author. Tel.: +31 306096872; fax: +31 306031204

E-mail address: d.m.s.ronden@differ.nl (D.M.S. Ronden).
}

(DFW) that is attached to the plasma facing side of the DSM offers neutron shielding for the GUPP/DSM contents.

The aim of the DSM configuration is to be able to extract it from the GUPP after which full access to the GUPP internals is offered. This task is foreseen to take place in the ITER hot cell facility (HCF), with the GUPP oriented vertically, so that the DSM can be handled by an overhead crane [2].

One of the main drivers behind the development of the GUPP is the minimization of down-time due to maintenance activity and of the variety of tools needed to perform maintenance. This paper discusses the RH compatibility analysis (RHCA) of the GUPP design as it was presented at the GUPP final design review at ITER 


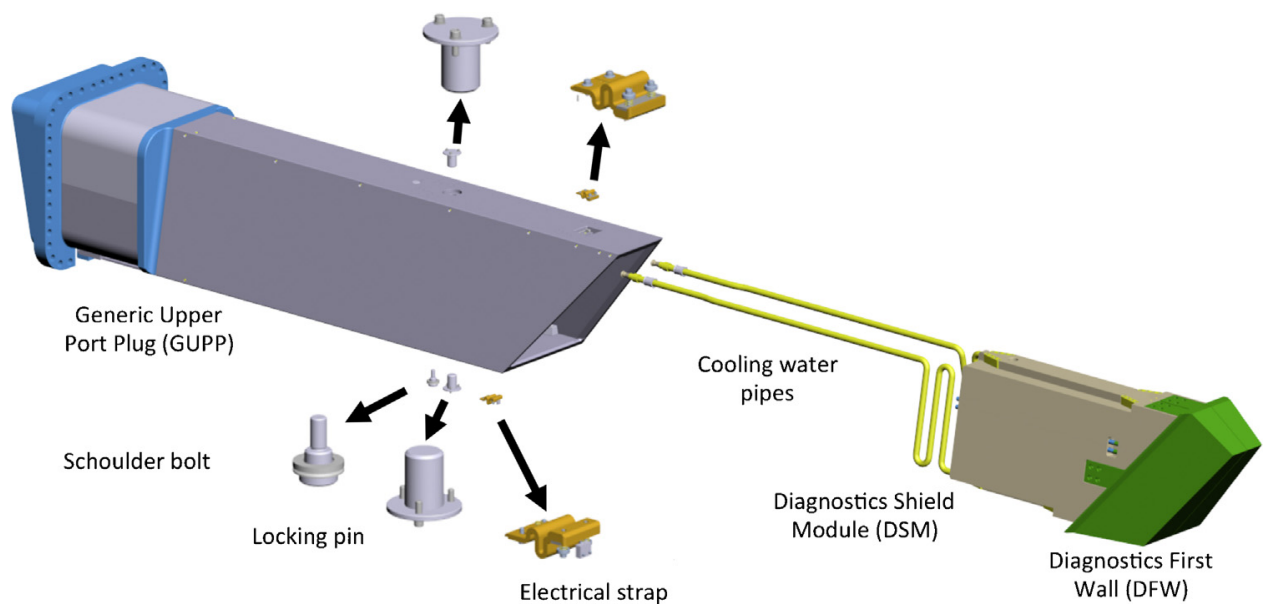

Fig. 1. Exploded view of the GUPP, highlighting all key elements.

IO, held on 24-25 June 2013. The RHCA was performed on the design of the generic upper port plug; this means that some aspects will differ between specific upper diagnostic port plugs. The pipe feedthroughs are discussed, but were not within the scope of this study.

\section{Design of GUPP DSM interface}

The GUPP design is described in [1-4]. Information relevant for the presentation of this study is given in this section. The full RHCA report is also available on the ITER database [5].

\subsection{DSM guidance}

Both the GUPP structure and the DSM are fitted with pads and rails that are used specifically for guiding the DSM to its final position during insertion before it is bolted to the GUPP structure. The pads and rails have tapered extremities to narrow down positioning accuracy to within $\pm 0.5 \mathrm{~mm}$. The pads and rails are highlighted in Figs. 2 and 3.

\subsection{DSM fixation}

The DSM is secured inside the GUPP by two locking pins - one on top and one at the bottom of the GUPP. A large shoulder bolt is used to anchor the DSM to the bottom wall of the GUPP. Each locking pin is secured by three bolts, while the shoulder bolt is a large M30 bolt that is mounted by a high-torque (hydraulic) bolting tool. The female thread for the shoulder bolt is integrated in one of the bottom positioning pads depicted in Fig. 3.

Neither the shoulder bolt, nor the locking pin fix the DSM rigidly to the GUPP structure; the stopper pads, together with the locking pins limit the movement to $0-0.25 \mathrm{~mm}$, while the shoulder bolts restrict poloidal movement of the DSM inside the GUPP to $0.25 \mathrm{~mm}$. In toroidal direction, the pads and rails leave $\pm 0.5 \mathrm{~mm}$ of play between the GUPP structure and the DSM.

\subsection{Electrical straps}

During a plasma disruption, strong eddy currents are generated in all conductive elements that feel the delta flux of the disrupting plasma. To prevent components from welding together due to arcs resulting from the eddy currents, $\mathrm{RH}$ compatible electrical straps are proposed that form a highly conductive electrical connection between the GUPP structure and the DSM (Fig. 4).
When the DSM is removed from the GUPP structure, the straps are unfastened first. In order to minimize task complexity, the strap was designed such that when loosened, it retracts into the GUPP outer wall through a pre-loaded spring and does not need to be disassembled. However further disassembly is possible in case the strap itself has to be replaced.

\subsection{Cooling pipe feedthrough}

The cooling pipes offer coolant to the DSM and run through the GUPP closure plate. They are welded from the ex-vessel side. To streamline the alignment of the pipes into the feedthroughs when the DSM is inserted, funnels are mounted on the in-vessel side (Fig. 5).

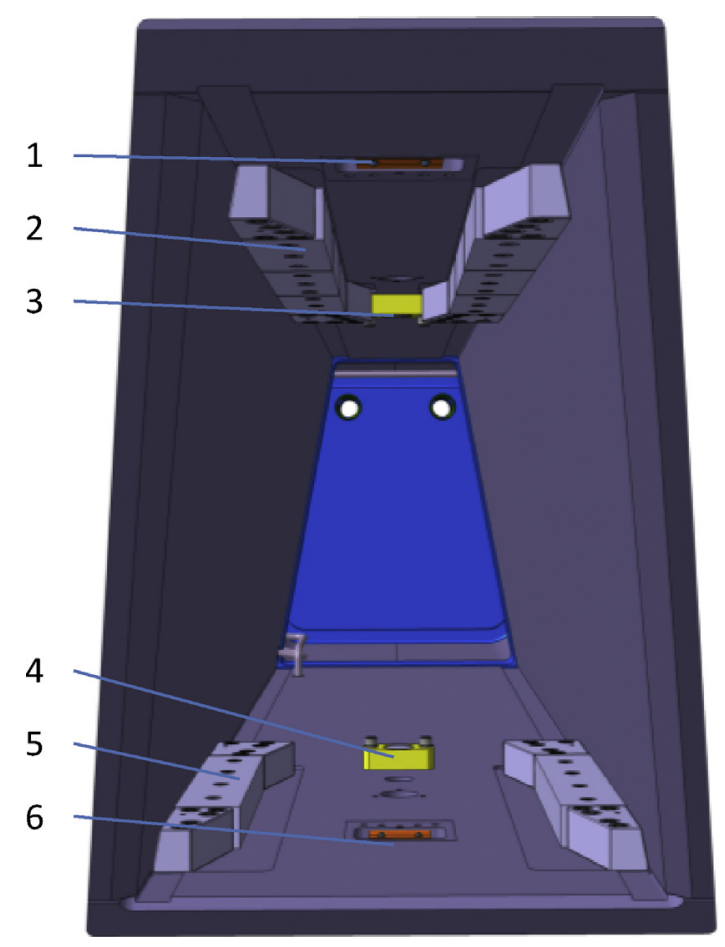

Fig. 2. Frontal view into the GUPP with the DSM and its fixtures removed. (1) Upper electrical strap; (2) upper rails; (3) upper axial stopper; (4) lower axial stopper; (5) lower rails; (6) lower electrical strap. 


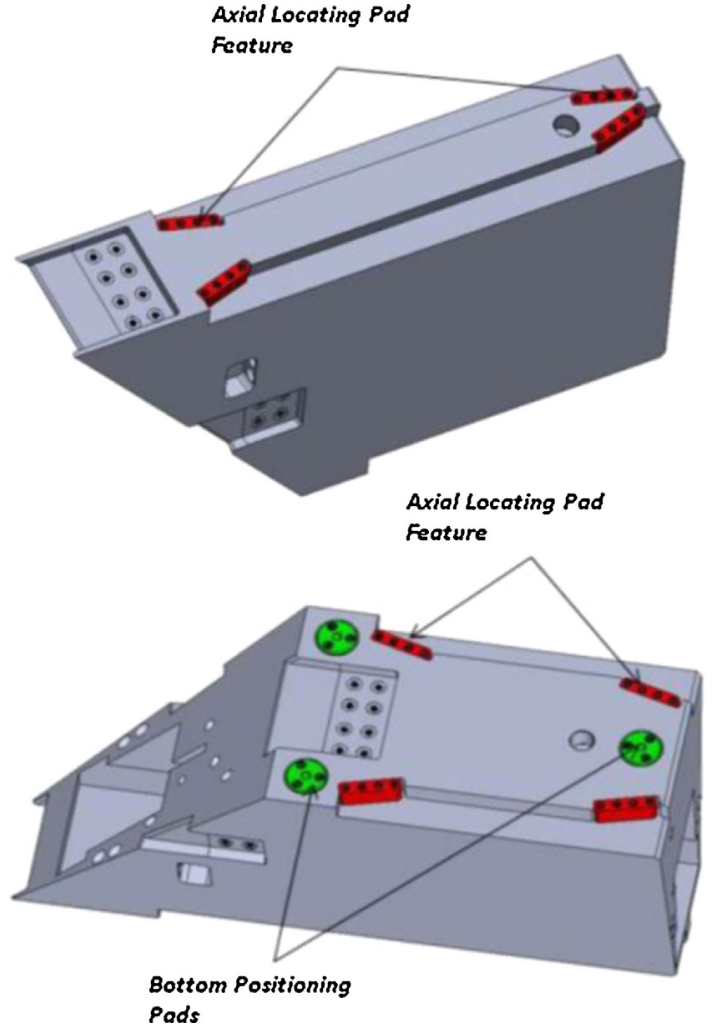

Fig. 3. Top and bottom view of the DSM, showing the pads that are guided by the GUPP rails.

\section{RHCA of the design}

The GUPP design was evaluated with respect to RH compatibility, and compared with the ITER RH Code of Practice [6]. Some GUPP design features appear to be fully RH compatible:
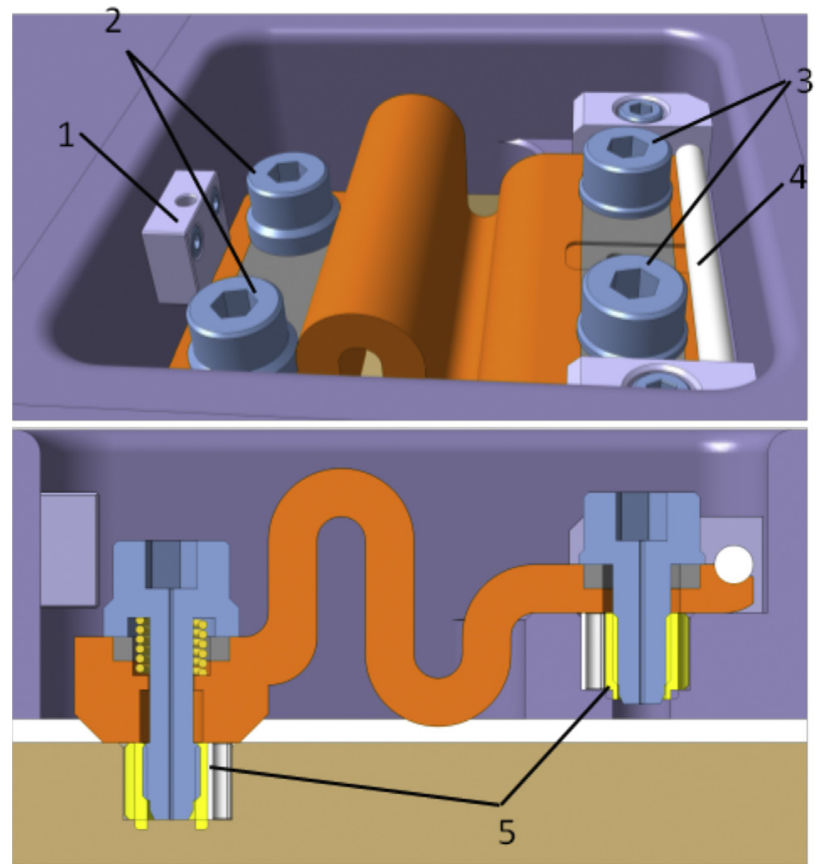

Fig. 4. The electrical straps with all key parts indicated; (1) stopper block; (2) captive pop-up bolts; (3) standard bolts; (4) hinge; (5) Spiralock inserts. Not shown is the spring that pushes the strap upwards, which is located between the two bolts to the right.

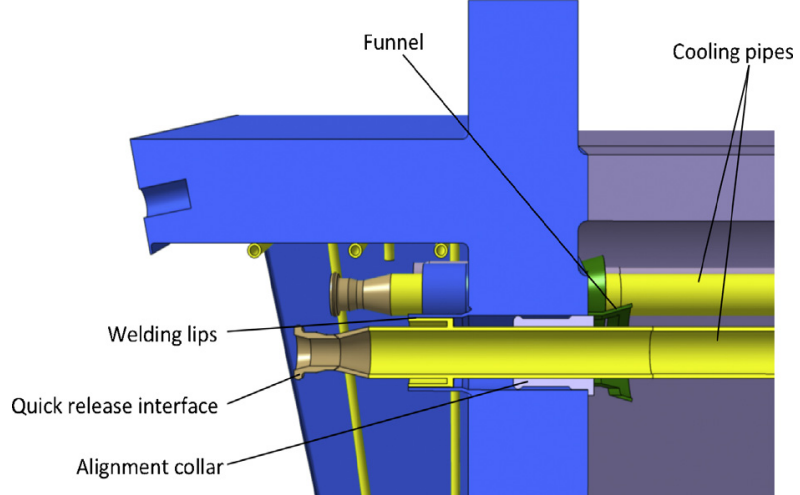

Fig. 5. Cross section of the GUPP closure plate, showing the layout of the cooling pipe feedthrough.

- Sliding rails, stopper pads and axial locating pads

- Electrical strap

- Shoulder bolt (with minor modifications)

The shoulder bolt is too large to make it a captive design and therefore it is proposed to integrate a tooling interface with a ball detent mechanism that anchors the bolt to the tool, similar to the mechanism found in replaceable socket wrenches.

The evaluation revealed a few GUPP design features with shortcomings. Alternatives were proposed to comply with $\mathrm{RH}$ requirements.

\subsection{Locking pin}

The locking pin should contain captive pop-up bolts and an attachment point for a $\mathrm{RH}$ gripping feature. Furthermore the tolerances in the locking pin design result in risk of jamming. A lead-in feature would make the task of inserting the pin into its slot less prone to jamming. Finally no special measures were taken for recovery operations. A central shaft for drill centering would suffice. Work on an updated locking pin design is ongoing.

\subsection{Tilting of the DSM}

The current design of the DSM allows for too much rotation during insertion into the GUPP resulting in hazardous situation for interfaces and components. This became apparent for the cooling pipes during the animation (Section 5). Each DSM has a specific payload and thus a specific centre of gravity. This centre of gravity need to be taken into account during hoisting as hoisted components will move there centre of gravity below the hoisting point, Ways to overcome this are either to restrict misalignment by extending the DSM guiding features, to introduce a rigid support frame on the back of the DSM, or to design an adjustable DSM crane interface to suspend the DSM perfectly vertical. The latter is difficult to check during the extraction procedure.

\section{RH tooling}

To perform the DSM replacement operation inside the HCF, a number of $\mathrm{RH}$ tools are needed that are currently not listed in the ITER RH Code of Practice [6].

\subsection{UPP vertical rotator device}

A large device capable of positioning the upper port plugs vertically. 


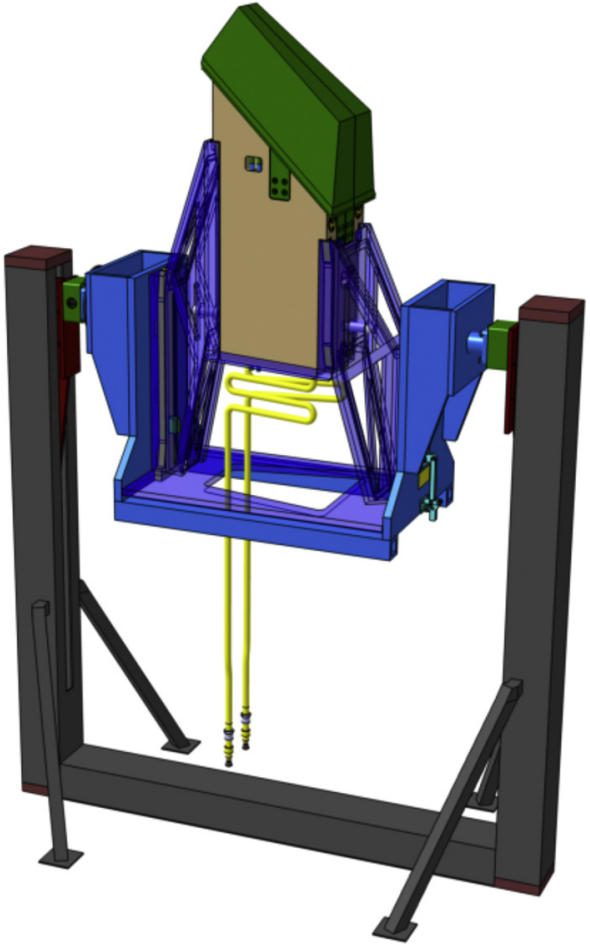

Fig. 6. Impression of the GEPP DSM handling device, containing an adapter piece and a GUPP DSM.

\subsection{Hydraulic bolting tool M30}

The hydraulic bolting tool listed in [6] can deliver $600 \mathrm{Nm}$ of torque, while for M30 bolts up to $2500 \mathrm{~N}$ m is needed. Integration of a ball detent mechanism is proposed.

\subsection{DSM storage/maintenance device}

A conceptual design already existed of the generic equatorial port plug (GEPP) DSM handling device. To limit storage space of the large variety of specific tools, it is proposed to use an adapter piece to make the GEPP-DSM handling device suitable for accepting GUPP DSM as well (Fig. 6).

\subsection{DFW hoisting tool}

The GUPP DSM will be hoisted and transported by the HCF overhead crane. In the development of the DFW [7], a pre-conceptual design of a DFW handling tool was proposed, which can also be used for lifting of the GUPP-DSM.

\subsection{General $R H$ tooling and facilities}

The lack of available plug handling tools in the IO $\mathrm{RH}$ tool database and delays in the design of the hot cell facility and its equipment make the assessment of RH compatibility challenging. The space availability inside the HCF for instance, is an ongoing task to be resolved soon. For a proper compatibility assessment, it should be clear what the plant design should be compatible with. The available ITER RH documentation offer guidelines, but should mature further before a final assessment can be made.

\section{Task simulation}

RH compatibility of the DSM replacement procedure is demonstrated by a simulation in a VR environment that has an

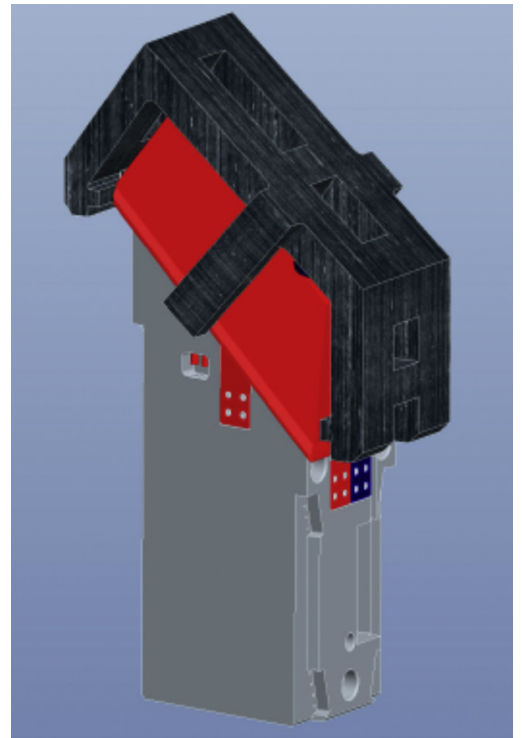

Fig. 7. Impression of the DFW/DSM handling device, mounted on top of the DFW.

incorporated physics engine [8]. This allows us to simulate collision dynamics, contact friction and the effects of accidental misalignment of components during assembly. A number of particular issues with the GUPP design became apparent from the simulation of the DSM replacement procedure.

In case that the DSM is lifted by the DFW handling device (Fig. 7), the assembly centre of gravity ( $\mathrm{CoG}$ ) should lie precisely below the hoisting point. When this is not the case, unspecified rotation of the assembly ensues, with unpredictable effects on the handling procedure. Aligning the CoG of the DSM is more challenging for the removal procedure as the neutral DSM orientation can only be measured when the DSM is suspended freely, while now it is still constrained inside the GUPP structure.

For insertion of the DSM, the cooling pipes are guided towards the holes in the closure plate by conical funnels. Despite the presence of funnels, the simulation showed that misalignment is possible as depicted in Fig. 8. CAD Measurements [5] verify that with the maximum angular misalignment of $\sim 0.7^{\circ}$ (DFW tilting downwards when GUPP is viewed from its horizontal orientation) between the GUPP and DSM, the axis-to-axis misalignment of the

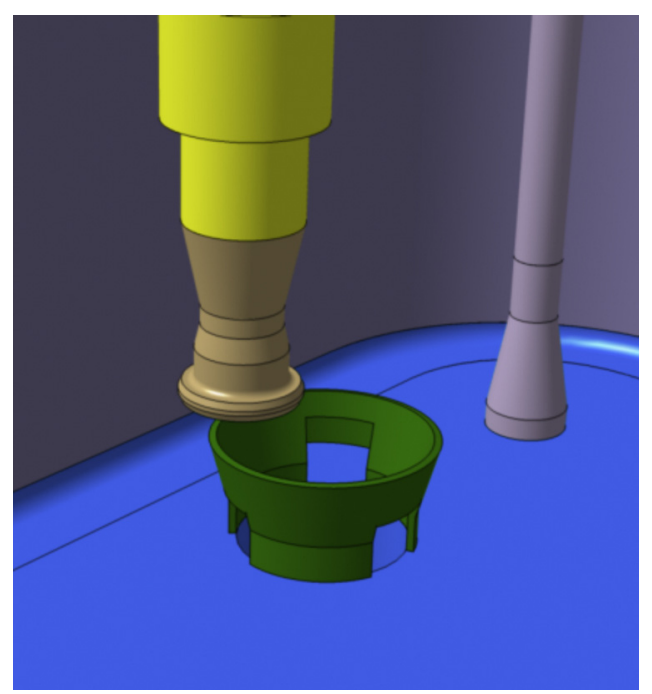

Fig. 8. Maximum angular misalignment of the DSM may lead to the cooling pipe colliding with the guiding funnel at the closure plate. 


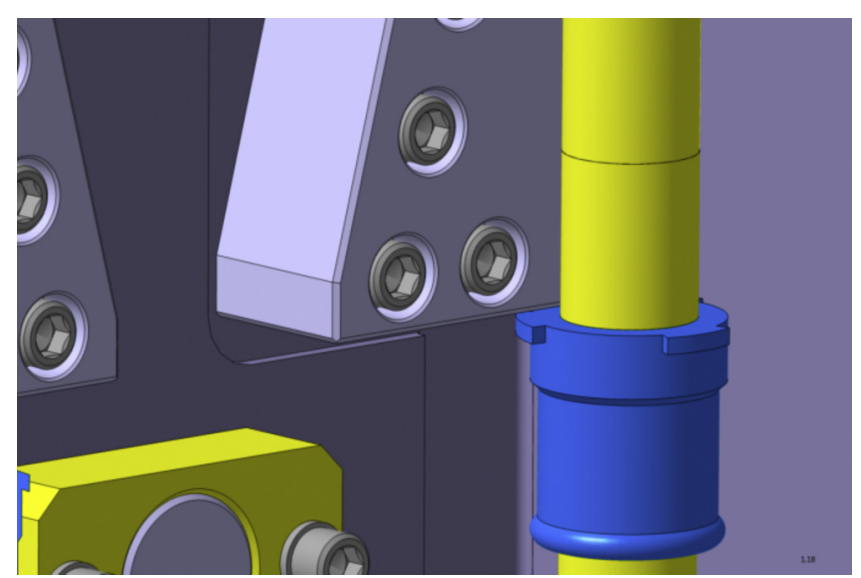

Fig. 9. Hooking of the cooling pipe collars behind the guiding rails will occur with moderate lateral $(30 \mathrm{~mm})$ or minute angular $\left(0.27-0.36^{\circ}\right)$ misalignments of the DSM relative to the GUPP structure.

pipe end at the funnel is $\sim 44 \mathrm{~mm}$. Although it may not be necessary for the funnel to catch the pipe end with maximum misalignment, it would make the design more robust if proper alignment is ensured by the design.

Due to inclusion of contact dynamics, the simulation of the task indicated an unexpected situation. A collision was found to occur between the cooling pipe collar and the GUPP upper guiding rails (Fig. 9). This is caused by an improperly located centre of gravity relative to the hoisting point, or when the crane is misaligned relative to the GUPP. Once the DSM is hoisted from the GUPP while the cooling pipes are still partially within the GUPP, the DSM can rotate more than the $\sim 0.7^{\circ}$, which will lead to the collision. The nominal distance between the collar and the rail is $26 \mathrm{~mm}$; in case of an angular misalignment between $0.27^{\circ}$ and $0.36^{\circ}$, the cooling pipe collar will snag behind the guiding rails. Greater misalignments will push the pipe itself against the rail, with a risk of bending the pipe. Since there are no visual cues on the outside to monitor such minute misalignments, nor any access holes for inspection cameras, these potential collisions are difficult to detect.

Several measures can prevent collisions between the GUPP rail and the cooling pipes. The collars could be fitted with conical edges to prevent hooking, although that would not prevent potential deformations to the pipes. A far more promising solution would be a support structure that is mounted to the back of the GUPP DSM. It can act as a protective cage surrounding components between the DSM and the closure plate. Effectively this extends the DSM outer shape, with the benefit that it restricts the rotation of the
DSM inside the GUPP during mounting. An additional advantage of this support structure is that it facilitates the gross alignment of DSM to the GUPP and DSM handing device.

\section{Summary and conclusions}

The RHCA of the GUPP has shown that some features of the design are fully $\mathrm{RH}$ compatible (sliding rails, stopper pads, axial locating pads, electrical strap and the shoulder bolt with minor adjustments), while others need some further modifications (locking pins and protection against DSM rotation during (dis)assembly).

The animation of the replacement task showed that there is a risk that tilting of the DSM results in appendages of the DSM hooking behind other features inside the GUPP structure. This can occur either during insertion, extraction or both. The offered solution is to extend guiding features on the back end of the DSM by the use of a support frame.

Conceptual tooling solutions are proposed to service and handle the DSM, while keeping the variety of tools to a minimum. This could further be optimized by standardization of interfaces. Moreover, it is suggested to expand the tooling database with regards to the range of tool sizes and the variety of (generic) plug handling tools. Determining the space availability inside the HCF is an ongoing task to be resolved soon.

\section{Acknowledgements}

This work was carried out within IO framework contract ITER/CT/6000000107. The views and opinions expressed herein do not necessarily reflect those of the ITER Organization.

\section{References}

[1] C.S. Pitcher, R. Barnsley, L. Bertalot, A. Encheva, R. Feder, J.P. Friconneau, et al. Port-based plasma diagnostic intrastructure on ITER, TOFE-2012, Fusion Sci. Technol. (2014) (in press).

[2] R. Feder, S. Pak, Technical Specifications of Diagnostic Shield Module Interfaces in the Common Upper Port Plug Structure, private communication.

[3] V.S. Udintsev, S. Pak, System Design Description (DDD) 55.U0 Up Port Systems, private communication.

[4] S. Pak, P. Maquet, Generic Diagnostic Port Plug Requirements, private communication.

[5] D.M.S. Ronden, V.S. Udintsev Remote Handling Compatibility Report for Generic Upper Port Plug RHCR-23-55.U0, private communication.

[6] R. Subramanian, J. Palmer, ITER Remote Handling Code of Practice, private communication.

[7] G.D. Loesser, V. Udintsev, J-M. Drevon, R. Feder, D. Johnson, P. Maquet, et al. Design Progress of ITER Diagnostic First Wall (SOFE-25), 2013, June.

[8] J. van Oosterhout, C.J.M. Heemskerk, J.F. Koning, D.M.S. Ronden, M. de Baar, Interactive Virtual Mock-ups for Remote Handling Compatibility Assessment of Heavy Components, this conference. 